\title{
Compact 1.5-GHz Intra-burst Repetition Rate Yb-doped All-PM-Fiber Laser System for Ablation-cooled Material Removal
}

\author{
Önder AKÇAALAN ${ }^{1}$, Hamit KALAYCIOĞLU ${ }^{1}$, Parviz ELAHI ${ }^{1}$, Petro DEMINSKYI ${ }^{1}$, F. Ö. İlday \\ 1.Department of Physics, Bilkent University, Ankara, Turkey. \\ 2. Department of Electrical Engineering, Bilkent University, Ankara, Turkey.
}

Femtosecond (fs) laser pulse sources have become increasingly popular in the last decade as a result of their practical features, such as insensitivity to environmental variations, versatile designs, high power outputs. However, much of the progress is with non-integrated specialty fibers, which involve some compromise on these practical features. Monolithic fiber chirped pulse amplification (CPA) systems are very attractive for industrial and scientific applications due to the features such as compactness, reliability and robustness. Although fs fiber laser systems are powerful technologies for material and tissue processing, limited ablation rates and high energy are drawbacks. Recently, we identified a new regime of laser -material interaction, ablation cooled material removal [1], where the repetition rate has to be high enough so that the targeted spot size cannot cool down substantially by heat conduction which scales down ablation threshold by several orders of magnitude and reduces thermal effects to the bulk of the target. Here, we demonstrate a compact all-PM-fiber laser amplifier system with an intra-burst repetition rate of $1.5 \mathrm{GHz}$ able to produce bursts ranging from 20-ns to 65 -ns duration with $20 \mu \mathrm{J}$ to $80 \mu \mathrm{J}$ total energy, respectively, and pulses with up to $1 \mu \mathrm{J}$ individual energy at burst repetition rates ranging from $25 \mathrm{kHz}$ to $200 \mathrm{kHz}$ (Fig. 1(a)). The seed signal is generated by a home-built all-normal dispersion oscillator with a spectrum centered at $1035 \mathrm{~nm}$ and 20-nm (FWHM), $100 \mathrm{~mW}$ output and $385 \mathrm{MHz}$ repetition rate (Fig. 1(b)). After the oscillator, rest of the system is built of polarization maintaining (PM) components and a single-mode pre-amplifier controls both dispersion and nonlinearity in the amplifier system. The pulses are stretched with a $110 \mathrm{~m}$-long fiber after this pre-amplifier and raised to a repetition rate of $1.5 \mathrm{GHz}$ by a multiplier. The signal is amplified again by a second single-mode pre-amplifier before converted into burstmode via an acousto-optic modulator (AOM). Finally, a forward-pumped double-clad power amplifier, built of PM 10/125 Yb 1200 DC (nLight) fiber and pumped by a 18-W wavelength stabilized diode, boosts the optical power. To compress the pulses, a pair of 1200 line/mm transmission gratings is preferred to denser gratings to limit third order dispersion (TOD). Further, fiber lengths are shortened as much as possible to minimize nonlinear effects including Raman scattering and thus the power conversion efficiency is relatively low, around $20 \%$ for the power amplifier. The autocorrelation measurement for the compressed pulses indicates a width of $\sim 250$ fs (Fig. 1(d)). The amplified output spectrum of FWHM of $14 \mathrm{~nm}$ is shown in (Fig. 1(c)).
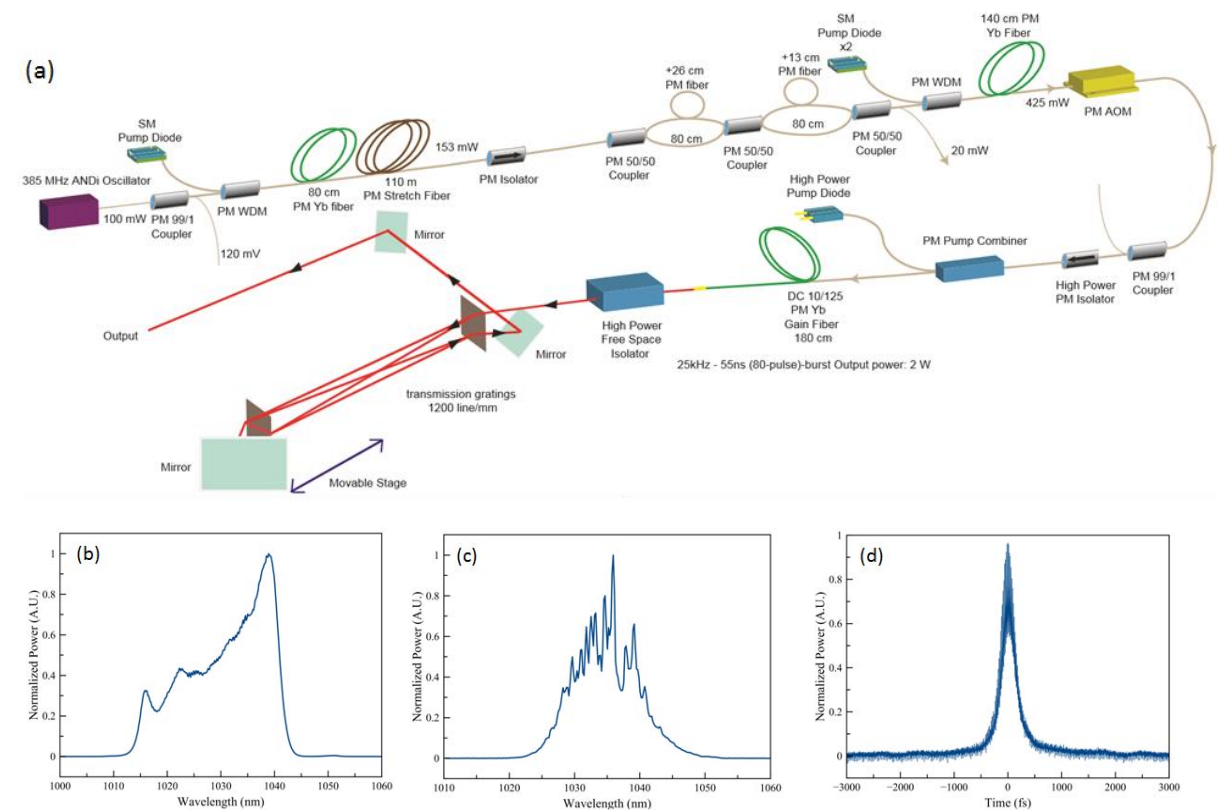

Fig. 1 (a) 1.5-GHz intra-burst repetition rate Yb-doped all-PM-fiber laser system setup. (b) The oscillator spectrum. Compressor output (c) spectrum and (d) pulse duration for $1 \mu \mathrm{J}$ individual pulse energy, 80 -pulse bursts at $25 \mathrm{kHz}$.

[1] C. Kerse, H. Kalaycioğlu, P. Elahi, B. Çetin, D. K. Kesim, Ö. Akçaalan, S. Yavaş, M. D. Aşık, B. Öktem, H. Hoogland, R. Holzwarth, and, F. Ö. Ilday, “Ablation-cooled material removal with ultrafast bursts of pulses," Nature 537, 84-88 (2016). 